\title{
THE DIFFERENCE ORLICZ SPACE OF ENTIRE SEQUENCE OF FUZZY NUMBERS
}

\author{
Nagarajan Subramanian And Ayhan Esi
}

ABSTRACT. In this paper we define and study the difference Orlicz space of entire sequence of fuzzy numbers. We study their different properties and statistical convergence in these spaces.

\section{Introduction}

The concept of fuzzy sets and fuzzy set operations were first introduced Zadeh [45] and subsequently several authors have discussed various aspects of the theory and applications of fuzzy sets such as fuzzy topological spaces, similarity relations and fuzzy orderings, fuzzy measures of fuzzy events, fuzzy mathematical programming.

In this paper we introduce and examine the concepts of Orlicz space of entire sequence of fuzzy numbers.

Let $D$ be the set of all bounded intervals $A=[A, \bar{A}]$ on the real line $\mathbb{R}$. For $A, B \in D$, define $A \leq B$ if and only if $\underline{A} \leq \underline{B}$ and $\bar{A} \leq \bar{B}, d(A, B)=$ $\max \{\underline{A}-\underline{B}, \bar{A}-\bar{B}\}$. Then it can be easily see that $d$ defines a metric $D$ (cf. [8]) and $(D, d)$ is a complete metric space.

A fuzzy number is a fuzzy subset of the real line $\mathbb{R}$ which is bounded, convex and normal. Let $L(\mathbb{R})$ denote the set of all fuzzy numbers which are upper semi continuous and have compact support, i.e., if $X \in L(\mathbb{R})$, then for any $\alpha \in[0,1], X^{\alpha}$ is compact, where

$$
X^{\alpha}= \begin{cases}t: X(t) \geq \alpha, & \text { if } 0<\alpha \leq 1, \\ t: X(t)>0, & \text { if } \alpha=0 .\end{cases}
$$

For each $0<\alpha \leq 1$, the $\alpha$-level set $X^{\alpha}$ is a nonempty compact subset of $\mathbb{R}$. The linear structure of $L(\mathbb{R})$ includes addition $X+Y$ and scalar multiplication $\lambda X(\lambda$ : scalar $)$ in terms of $\alpha$-level sets, by $[X+Y]^{\alpha}=[X]^{\alpha}+[Y]^{\alpha}$ and $[\lambda X]^{\alpha}=$ $\lambda[X]^{\alpha}$ for each $0 \leq \alpha \leq 1$. Define a map $\bar{d}: L(\mathbb{R}) \times L(\mathbb{R}) \rightarrow \mathbb{R}$ by $\bar{d}(X, Y)=$ $\sup _{0 \leq \alpha \leq 1} d\left(X^{\alpha}, Y^{\alpha}\right)$. For $X, Y \in L(\mathbb{R})$ define $X \leq Y$ if and only if $X^{\alpha} \leq Y^{\alpha}$

Received April 30, 2009.

2000 Mathematics Subject Classification. 40A05, 40C05, 40 D05.

Key words and phrases. fuzzy numbers, Orlicz space, entire sequence, analytic sequence, difference sequence. 
for any $\alpha \in[0,1]$. It is shown that $(L(\mathbb{R}), \bar{d})$ is a complete metric space (cf. [26]).

A complex sequence, whose $k^{t h}$ terms is $x_{k}$ is denoted by $\left\{x_{k}\right\}$ or simply $x$. Let $\phi$ be the set of all finite sequences. Let $\ell_{\infty}, c, c_{0}$ be the sequence spaces of bounded, convergent and null sequences $x=\left(x_{k}\right)$ respectively. In respect of $\ell_{\infty}, c, c_{0}$ we have $\|x\|=\sup _{k}\left|x_{k}\right|$, where $x=\left(x_{k}\right) \in c_{0} \subset c \subset \ell_{\infty}$. A sequence $x=\left\{x_{k}\right\}$ is said to be analytic if $\sup _{k}\left|x_{k}\right|^{1 / k}<\infty$. The vector space of all analytic sequences will be denoted by $\Lambda$. A sequence $x$ is called entire sequence if $\lim _{k \rightarrow \infty}\left|x_{k}\right|^{1 / k}=0$. The vector space of all entire sequences will be denoted by $\Gamma$. Orlicz [35] used the idea of Orlicz function to construct the space $\left(L^{M}\right)$. Lindenstrauss and Tzafriri [23] investigated Orlicz sequence spaces in more detail, and they proved that every Orlicz sequence space $\ell_{M}$ contains a subspace isomorphic to $\ell_{p}(1 \leq p<\infty)$. Subsequently different classes of sequence spaces defined by Parashar and Choudhary [36], Mursaleen et al. [29], Bektas and Altin [4], Tripathy et al. [42], Rao and Subramanian [37] and many others. The Orlicz sequence spaces are the special cases of Orlicz spaces studied in [20].

Recall ([35], [20]) an Orlicz function is a function $M:[0, \infty) \rightarrow[0, \infty)$ which is continuous, non-decreasing and convex with $M(0)=0, M(x)>0$ for $x>0$ and $M(x) \rightarrow \infty$ as $x \rightarrow \infty$. If convexity of Orlicz function $M$ is replaced by $M(x+y) \leq M(x)+M(y)$, then this function is called a modulus function, introduced by Nakano [30] and further discussed by Ruckle [39] and Maddox [25] and many others.

An Orlicz function $M$ is said to satisfy $\Delta_{2}$-condition for all values of $u$, if there exists a constant $K>0$ such that $M(2 u) \leq K M(u)(u \geq 0)$. The $\Delta_{2^{-}}$ condition is equivalent to $M(\ell u) \leq K \ell M(u)$ for all values of $u$ and for $\ell>1$. Lindenstrauss and Tzafriri [23] used the idea of Orlicz function to construct Orlicz sequence space

$$
\ell_{M}=\left\{x \in w: \sum_{k=1}^{\infty} M\left(\frac{\left|x_{k}\right|}{\rho}\right)<\infty \text { for some } \rho>0\right\}
$$

The space $\ell_{M}$ with the norm

$$
\|x\|=\inf \left\{\rho>0: \sum_{k=1}^{\infty} M\left(\frac{\left|x_{k}\right|}{\rho}\right) \leq 1\right\}
$$

becomes a Banach space which is called an Orlicz sequence space. For $M(t)=$ $t^{p}, 1 \leq p<\infty$, the space $\ell_{M}$ coincide with the classical sequence space $\ell_{p}$. Given a sequence $x=\left\{x_{k}\right\}$, its $n^{t h}$ section is the sequence $x^{(n)}=\left\{x_{1}, x_{2}, \ldots, x_{n}, 0,0\right.$, $\ldots\}, \delta^{(n)}=(0,0, \ldots, 1,0,0, \ldots), 1$ in the $n^{t h}$ place and zero's else where. 


\section{Definitions and prelimiaries}

Let $w$ denote the set of all fuzzy complex sequences $x=\left(x_{k}\right)_{k=1}^{\infty}$, and $M$ be an Orlicz fucntion, or a modulus function. consider

$$
\begin{aligned}
& \Gamma_{M}=\left\{x \in w: \lim _{k \rightarrow \infty}\left(M\left(\frac{\left|x_{k}\right|^{1 / k}}{\rho}\right)\right)=0 \text { for some } \rho>0\right) \text { and } \\
& \Lambda_{M}=\left\{x \in w: \sup _{k}\left(M\left(\frac{\left|x_{k}\right|^{1 / k}}{\rho}\right)\right)<\infty \text { for some } \rho>0\right) .
\end{aligned}
$$

The space $\Gamma_{M}$ and $\Lambda_{M}$ is a metric space with the metric

$$
d(x, y)=\inf \left\{\rho>0: \sup _{k}\left(M\left(\frac{\left|x_{k}-y_{k}\right|^{1 / k}}{\rho}\right)\right) \leq 1\right\}
$$

for all $x=\left\{x_{k}\right\}$ and $y=\left\{y_{k}\right\}$ in $\Gamma_{M}$.

In this paper we define Orlicz space of entire sequence of fuzzy numbers by using regular matrices $A=\left(a_{n k}\right)(n k=1,2,3, \ldots)$. By the regularity of $A$ we mean that the matrix which transform convergent sequence into a convergent sequence leaving the limit (cf. Maddox [24]); we prove that these spaces are complete paranormed spaces. If $E$ is a linear space over the filed $C$, then a paranorm on $E$ is a function $g: E \rightarrow \mathbb{R}$ which satisfies the following axioms; for $X, Y \in E$,

(P.1) $g(\theta)=0$,

(P.2) $g(X) \geq 0$ for all $X \in E$,

(P.3) $g(-X)=g(X)$ for all $X \in E$,

(P.4) $g(X+Y) \leq g(X)+g(Y)$ for all $X, Y \in E$,

(P.5) If $\left(\lambda_{n}\right)$ is a sequence of scalars with $\lambda_{n} \rightarrow \lambda(n \rightarrow \infty)$ and $\left(X_{n}\right)$ is a sequence of the elements of $E$ with $X_{n} \rightarrow X$ imply $\lambda_{n} X_{n} \rightarrow \lambda X$, where $\lambda_{n} \lambda \in C$ and $X_{n}, X \in E$; In other words $\left|\lambda_{n}-\lambda\right| \rightarrow 0, g\left(X_{n}-X\right) \rightarrow 0$ imply $g\left(\lambda_{n} X_{n}-\lambda X\right) \rightarrow 0(n \rightarrow \infty)$.

A paranormed space is a linear space $E$ with a paranorm $g$ and is written as $(E, g)$ we now give the following new definitions which will be needed in the sequel.

Definition 2.1. A sequence $X=\left(X_{k}\right)$ of fuzzy number is a function $X$ from the set $N$ of natural numbers into $L(\mathbb{R})$. The fuzzy number $X_{n}$ denotes the value of the function $n \in N$ and is called $n^{t h}$ term of the sequence. We denote by $W(F)$ the set of all sequences $X=\left(X_{k}\right)$ of fuzzy numbers.

Definition 2.2. Let $X=\left(X_{k}\right)$ be a sequence of fuzzy numbers. Then the sequence $X=\left(X_{k}\right)$ of fuzzy numbers is said to be Orlicz space of entire sequence of fuzzy numbers convergent to zero, written as $\left(M\left(\frac{\left|X_{k}\right|^{1 / k}}{\rho}\right)\right) \rightarrow 0$ as $k \rightarrow \infty$, for some arbitrarily fixed $\rho>0$ and is defined by

$$
\left[\bar{d}\left(M\left(\frac{\left|X_{k}\right|^{1 / k}}{\rho}\right)\right) \rightarrow 0 \text { as } k \rightarrow \infty\right]
$$

is denoted by $\Gamma_{M}(F)$, with $M$ being a modulus function. 
Definition 2.3. Let $X=\left(X_{k}\right)$ be a sequence of fuzzy numbers. Then the sequence $X=\left(X_{k}\right)$ of fuzzy numbers is said to be Orlicz space of analytic sequence if the set $\left\{\left(M\left(\frac{\left|X_{k}\right|^{1 / k}}{\rho}\right)\right): k \in N\right\}$ of fuzzy numbers. We denote $\Lambda_{M}(F)$ the set of all analytic sequence of fuzzy number.

\section{Orlicz space of entire sequence of fuzzy numbers}

In this paper we define the following

$$
\begin{aligned}
& \Gamma_{M}[F, p]=\left\{X=\left(X_{k}\right): \frac{1}{n} \sum_{k=1}^{n}\left[\bar{d}\left(M\left(\frac{\left|X_{k}\right|^{1 / k}}{\rho}\right), 0\right)\right]^{p_{k}} \rightarrow 0 \text { as } k \rightarrow \infty\right\}, \\
& \Lambda_{M}[F, p]=\left\{X=\left(X_{k}\right): \sup _{n} \frac{1}{n} \sum_{k=1}^{n}\left[\bar{d}\left(M\left(\frac{\left|X_{k}\right|^{1 / k}}{\rho}\right), 0\right)\right]^{p_{k}}<\infty\right\} \text { and }
\end{aligned}
$$
call them respectively the spaces of sequences of fuzzy numbers which are strongly Orlicz space of entire to zero and strongly analytic.

A metric $\bar{d}$ on $L(\mathbb{R})$ is said to be a translation invariant if $\bar{d}(X+Z, Y+Z)=$ $\bar{d}(X, Y)$ for $X, Y, Z \in L(\mathbb{R})$.

In this paper we define and study the difference Orlicz space of entire sequence of fuzzy numbers. The idea of difference sequences for real numbers was first introduced by Kizmaz [19].

\section{Difference Orlicz space of entire sequence of fuzzy numbers}

Let $X=\left(X_{k}\right)$ be a sequence of fuzzy numbers. Write $\Delta X=\left(X_{k}-X_{k+1}\right)$ $(k=1,2,3, \ldots)$. We define the following spaces of difference sequences of fuzzy numbers:

$$
\begin{aligned}
& \Gamma_{M}[F, A, p, \Delta]=\left\{X=\left(X_{k}\right) \in w(F): \Delta X \in \Gamma_{M}(F, A, p)\right\}, \\
& \Lambda_{M}[F, A, p, \Delta]=\left\{X=\left(X_{k}\right) \in w(F): \Delta X \in \Lambda_{M}(F, A, p)\right\} .
\end{aligned}
$$

Note that $\Gamma_{M}(F, A, p) \subset \Gamma_{M}[F, A, p, \Delta]$ and $\Lambda_{M}(F, A, p) \subset \Lambda_{M}[F, A, p, \Delta]$. We prove the following results:

Proposition 4.1. If $\bar{d}$ is a translation invariant metric on $L(\mathbb{R})$, then

(i) $\bar{d}(\Delta X+\Delta Y, 0) \leq \bar{d}(\Delta X, 0)+\bar{d}(\Delta Y, 0)$,

(ii) $\bar{d}(\lambda \Delta X, 0) \leq|\lambda| \bar{d}(\Delta X, 0),|\lambda|>1$.

Theorem 4.2. $\Gamma_{M}(F, p, \Delta)$ are complete metric spaces with the metric is given by

$$
\rho(X, Y)=\sup _{n}\left[\frac{1}{n} \bar{d}\left(M\left(\frac{\left|\Delta X_{n}+\Delta Y_{n}\right|^{1 / n}}{\rho}\right), 0\right)\right]^{p},
$$

where $X=\left(\Delta X_{n}\right)$ and $Y=\left(\Delta Y_{n}\right)$ are the difference sequences of fuzzy numbers. 
Proof. It is easy to show that these are metric spaces. We will show the completeness. Let $\left\{X^{(m)}\right\}$ be a Cauchy sequence in $\Gamma_{M}(F, p, \Delta)$. Then

$$
\left(M\left(\frac{\left|\Delta X_{n}^{(i)}\right|^{1 / n}}{\rho}\right)\right)
$$

will be a Cauchy sequence in $\Gamma_{M}(F, p, \Delta)$. Therefore for each $n$, then

$$
\left(M\left(\frac{\left|\Delta X_{n}^{(i)}\right|^{1 / n}}{\rho}\right)\right)
$$

is a Cauchy sequence in $L(\mathbb{R})$. Since $L(\mathbb{R})$ is complete,

$$
\left(\frac{1}{n}\left(M\left(\frac{\left|\Delta X_{n}^{(i)}\right|^{1 / n}}{\rho}\right)\right)\right) \rightarrow 0 \text { as } i \rightarrow \infty
$$

put $\Delta X=\left(\Delta X_{n}\right)$, since $\left(M\left(\frac{\left|\Delta X_{n}^{(i)}\right|^{1 / n}}{\rho}\right)\right)$ is a Cauchy sequence in $\Gamma_{M}(F, p, \Delta)$ there exists $n_{0} \in N$ such that for all $i$.

$$
\left(\frac{1}{n} \bar{d}\left(M\left(\frac{\left|\Delta X_{n}^{(i)}-\Delta X_{n}^{(j)}\right|^{1 / n}}{\rho}\right), 0\right)\right)^{p} \rightarrow 0 \text { as } n \rightarrow \infty .
$$

Let $j \rightarrow \infty$ we get (5) that

$$
\left(\frac{1}{n} \bar{d}\left(M\left(\frac{\left|\Delta X_{n}^{(i)}-\Delta X_{n}\right|^{1 / n}}{\rho}\right), 0\right)\right)^{p} \rightarrow 0 \text { as } n \rightarrow \infty .
$$

Therefore $\left(M\left(\frac{\left|\Delta X_{n}^{(i)}\right|^{1 / n}}{\rho}\right)\right) \rightarrow 0$ as $i \rightarrow \infty$. Now we have to show that $X \in$ $\Gamma_{M}(F, p, \Delta)$. Since $\Delta X^{(i)} \in \Gamma_{M}(F, p, \Delta)$, there exists $\Delta X_{0}^{(i)} \in L(\mathbb{R})$ such that

$$
\left(\frac{1}{n} \bar{d}\left(M\left(\frac{\left|\Delta X_{n}^{(i)}-\Delta X_{0}^{(i)}\right|^{1 / n}}{\rho}\right), 0\right)\right)^{p} \rightarrow 0 \text { as } n \rightarrow \infty
$$

$$
\begin{aligned}
& \text { Hence } \\
& \left(\frac{1}{n} \bar{d}\left(M\left(\frac{\left|\Delta X_{n}^{(i)}-\Delta X_{0}^{(j)}\right|^{1 / n}}{\rho}\right), 0\right)\right)^{p} \leq\left(\frac{1}{n} \bar{d}\left(M\left(\frac{\left|\Delta X_{n}^{(i)}-\Delta X_{0}^{(j)}\right|^{1 / n}}{\rho}\right), 0\right)\right)^{p} \\
& +\left(\frac{1}{n} \bar{d}\left(M\left(\frac{\left|\Delta X_{n}^{(i)}-\Delta X_{0}^{(i)}\right|^{1 / n}}{\rho}\right), 0\right)\right)^{p}+\left(\frac{1}{n} \bar{d}\left(M\left(\frac{\left|\Delta X_{n}^{(i)}-\Delta X_{0}^{(j)}\right|^{1 / n}}{\rho}\right), 0\right)\right)^{p}
\end{aligned}
$$


$\rightarrow 0$ as $n \rightarrow \infty$ by (5) and (7). Thus $\left(M\left(\frac{\left|\Delta X_{n}^{(i)}\right|^{1 / n}}{\rho}\right), 0\right)$ is a Cauchy sequence in $L(\mathbb{R})$. Since $L(\mathbb{R})$ is complete, there exists $\Delta X_{0} \in L(\mathbb{R})$ such that

$$
\left(\frac{1}{n} \bar{d}\left(M\left(\frac{\left|\Delta X_{0}^{(i)}-\Delta X_{0}\right|^{1 / n}}{\rho}\right), 0\right)\right)^{p} \rightarrow 0 \text { as } n \rightarrow \infty .
$$

Therefore by (6), (7) and (8) we have

$$
\begin{aligned}
& \left(\frac{1}{n} \bar{d}\left(M\left(\frac{\left|\Delta X_{n}-\Delta X_{0}\right|^{1 / n}}{\rho}\right), 0\right)\right)^{p} \leq\left(\frac{1}{n} \bar{d}\left(M\left(\frac{\left|\Delta X_{n}^{(i)}-\Delta X_{n}\right|^{1 / n}}{\rho}\right), 0\right)\right)^{p}+ \\
& \left(\frac{1}{n} \bar{d}\left(M\left(\frac{\left|\Delta X_{n}^{(i)}-\Delta X_{0}^{(i)}\right|^{1 / n}}{\rho}\right), 0\right)\right)^{p}+\left(\frac{1}{n} \bar{d}\left(M\left(\frac{\left|\Delta X_{0}^{(i)}-\Delta X_{0}\right|^{1 / n}}{\rho}\right), 0\right)\right)^{p} \rightarrow
\end{aligned}
$$

0 as $n \rightarrow \infty$. This implies that $X=\left(X_{k}\right) \in \Gamma_{M}(F, p, \Delta)$. Therefore $\Gamma_{M}(F, p, \Delta)$ is complete. This completes the proof.

Theorem 4.3. If $\bar{d}$ is a transition invariant metric and $M$ is a modulus function, then $\Gamma_{M}(F, p, \Delta)$ are linear spaces over the complex numbers $C$.

Proof. It is easy. Therefore omit the proof.

\section{Main results}

Theorem 5.1. $\Gamma_{M}(F, A, p, \Delta)$ and $\Lambda_{M}(F, A, p, \Delta)\left(\right.$ inf $\left.p_{k}>0\right)$ are complete with respect to the topology generated by the paranorm $h$ is defined by (9)

$h(X)=\sup _{n}\left(\sum_{k} a_{n k}\left[\bar{d}\left(M\left(\frac{\left|\Delta X_{k}\right|^{1 / k}}{\rho}\right), 0\right)\right]^{p_{k}}\right),\left|\sum_{k} a_{n k}\right|^{p_{k}} \rightarrow 0$ as $k \rightarrow \infty$, where $\bar{d}$ is a translation invariant and $X=\left(X_{k}\right)$ be a sequence of fuzzy numbers.

Proof. Let $\left(X^{(s)}\right)$ be a Cauchy sequence in $\Gamma_{M}(F, A, p, \Delta)$. Then

$$
\left[\bar{d}\left(M\left(\frac{\left|\Delta X^{s}-\Delta X^{t}\right|^{1 / k}}{\rho}\right), 0\right)\right] \rightarrow 0 \text { as } s, t \rightarrow \infty
$$

that is

(10)

$$
\left(\sum_{k} a_{n k}\left[\bar{d}\left(M\left(\frac{\left|\Delta X_{k}^{(s)}-\Delta X_{k}^{(t)}\right|^{1 / k}}{\rho}\right), 0\right)\right]\right) \rightarrow 0 \text { as } s, t \rightarrow \infty \text { for all } k \text {. }
$$

Hence

$$
\left[\bar{d}\left(M\left(\frac{\left|\Delta X_{k}^{(s)}-\Delta X_{k}^{(t)}\right|^{1 / k}}{\rho}\right), 0\right)\right] \rightarrow 0 \text { as } s, t \rightarrow \infty \text { for all } k
$$


which implies that $\left[\bar{d}\left(M\left(\frac{\left|\Delta X_{k}^{(s)}\right|^{1 / k}}{\rho}\right), 0\right)\right]$ is a Cauchy sequence $C$ for each $k$ and so there exists $Y=\left(\Delta Y_{k}\right)$ such that $\left[\bar{d}\left(M\left(\frac{\left|\Delta X_{k}^{(s)}\right|^{1 / k}}{\rho}\right), 0\right)\right] \rightarrow \Delta Y_{k}$ as $s \rightarrow \infty$ for each $k$. Now, from (10) we have, for $\epsilon>0$, there exists natural number $N$ such that

$$
\left(\sum_{k} a_{n k}\left[\bar{d}\left(M\left(\frac{\left|\Delta X_{k}^{(s)}-\Delta X_{k}^{(t)}\right|^{1 / k}}{\rho}\right), 0\right)\right]\right)<\epsilon
$$

for $s, t>N$ and for all $n$. Hence, for any fixed natural numbers $m$, we have from (11)

$$
\left(\sum_{k \leq m} a_{n k}\left[\bar{d}\left(M\left(\frac{\left|\Delta X_{k}^{(s)}-\Delta Y_{k}^{(t)}\right|^{1 / k}}{\rho}\right), 0\right)\right]\right)<\epsilon \text { for } s, t>N \text { for all } n .
$$

Now fix $s>N$ and let $t \rightarrow \infty$. Then from (11) we have

$$
\left(\sum_{k \leq m} a_{n k}\left[\bar{d}\left(M\left(\frac{\left|\Delta X_{k}^{(s)}-\Delta Y_{k}\right|^{1 / k}}{\rho}\right), 0\right)\right]\right)<\epsilon \text { for } s>N \text { for all } n \text {. }
$$

Since this is valid for any natural number $m$, we have

$$
\left(\sum_{k \leq m} a_{n k}\left[\bar{d}\left(M\left(\frac{\left|\Delta X_{k}^{(s)}-\Delta Y_{k}\right|^{1 / k}}{\rho}\right), 0\right)\right]\right)<\epsilon \text { for } s>N \text { for all } n,
$$

that is

$$
\left[\bar{d}\left(M\left(\frac{\left|\Delta X^{(s)}-\Delta Y\right|^{1 / k}}{\rho}\right), 0\right)\right] \rightarrow 0 \text { as } s \rightarrow \infty,
$$

and thus $\left[\bar{d}\left(M\left(\frac{\left|\Delta X^{(s)}\right|^{1 / k}}{\rho}\right), 0\right)\right] \rightarrow \Delta Y$ as $s \rightarrow \infty$, and thus

$$
\left[\bar{d}\left(M\left(\frac{\left|\Delta X^{(s)}-\Delta Y\right|^{1 / k}}{\rho}\right), 0\right)\right] \in \Gamma_{M}(F, A, p, \Delta),
$$

also writing $\Delta Y=\left[\bar{d}\left(M\left(\frac{\left|\Delta Y-\Delta X^{(s)}\right|^{1 / k}+\left|\Delta X^{(s)}\right|^{1 / k}}{\rho}\right), 0\right)\right]$, we have by linearity of $\Gamma_{M}(F, A, p, \Delta) \Delta Y \in \Gamma_{M}(F, A, p, \Delta)$; hence $\Gamma_{M}(F, A, p, \Delta)$ is complete. The completeness of $\Lambda_{M}(F, A, p, \Delta)$ can be similarly obtained. 
Theorem 5.2. Let $X=\left(X_{k}\right)$ be a sequence of fuzzy numbers and $\bar{d}$ be a translation invariant. Let $A=\left(a_{n k}\right)(n, k=1,2,3, \ldots)$ be an infinite matrix with complex entries. Then $A \in\left(\Gamma_{M}(F, A, p): \Gamma_{M}(F, A, p, \Delta)\right)$ if and only if given $\epsilon>0$ there exists $M=M(\epsilon)>0$ such that

$$
\left|a_{n k}-a_{n+1, k}\right|<\epsilon^{n} M^{k} \quad(n, k=1,2,3, \ldots) .
$$

Proof. Let $X=\left(X_{k}\right) \in \Gamma(F, A, p)$ and let

$$
Y_{n}=\left(\sum_{k=1}^{\infty} a_{n k} \bar{d}\left(M\left(\frac{\left|X_{k}\right|^{1 / k}}{\rho}\right), 0\right)^{p}\right)(n=1,2,3, \ldots),
$$

so that $\Delta Y_{n}=\sum_{k=1}^{\infty}\left(a_{n k}-a_{n+1, k}\right)\left(\bar{d}\left(M\left(\frac{\left|X_{k}\right|^{1 / k}}{\rho}\right), 0\right)^{p}\right)$. Then $\left(\Delta Y_{n}\right) \in$ $\Gamma_{M}(F, A, p)$ if and only if given any $\epsilon>0$ there exists $M=M(\epsilon)>0$ such that $\left|a_{n k}-a_{n+1, k}\right|<\epsilon^{n} M^{k}$ by using Theorem 4 of [38]. Now $\left(\Delta Y_{n}\right) \in \Gamma_{M}(F, A, p)$ if and only if $\left(Y_{n}\right) \in \Gamma_{M}(F, A, p, \Delta)$. Thus $A \in\left(\Gamma_{M}(F, A, p): \Gamma_{M}(F, A, p, \Delta)\right)$ if and only if the condition holds. This completes the proof.

Theorem 5.3. Let $X=\left(X_{k}\right)$ be a sequence of fuzzy numbers and $\bar{d}$ be a translation invariant. Let $A=\left(a_{n k}\right)$ transform $\Gamma_{M}(F, A, p)$ into $\Gamma_{M}(F, A, p, \Delta)$. Then $\lim _{n \rightarrow \infty}\left(a_{n k}-a_{n+1, k}\right) q^{n}=0$ for all integers $q>0$ and each fixed $k=1,2,3, \ldots$.

Proof. Let $Y_{n}=\left(\sum_{k=1}^{\infty} a_{n k} \bar{d}\left(M\left(\frac{\left|X_{k}\right|^{1 / k}}{\rho}\right), 0\right)^{p}\right) \quad(n=1,2,3, \ldots)$ formally. Let $\left(X_{k}\right) \in \Gamma_{M}(F, A, p)$ and $\left(Y_{n}\right) \in \Gamma_{M}(F, A, p, \Delta)$. But then

$$
\begin{aligned}
\left(\Delta Y_{n}\right) & \in \Gamma_{M}(F, A, p), \\
\Delta Y_{n} & =\left(\sum_{k=1}^{\infty}\left(a_{n k}-a_{n+1, k}\right) \bar{d}\left(M\left(\frac{\left|X_{k}\right|^{1 / k}}{\rho}\right), 0\right)^{p}\right)(n=1,2,3, \ldots) .
\end{aligned}
$$

Take $\left(X_{k}\right)=\delta^{k}=(0,0,0, \ldots, 1,0,0, \ldots), 1$ in the $k^{t h}$ place and zero's elsewhere. Then $\left(X_{k}\right) \in \Gamma_{M}(F, A, p)$. We have $\Delta Y_{n}=a_{n k}-a_{n+1, k}$. But $\left(\Delta Y_{n}\right) \in$ $\Gamma_{M}(F, A, p)$. Hence $\sum_{k=1}^{\infty}\left|a_{n k}-a_{n+1, k}\right| q^{n}<\infty$ for every positive integer $q$. In particular $\lim _{n \rightarrow \infty}\left(a_{n k}-a_{n+1, k}\right) q^{n}=0$ for all integers $q$ and each fixed $k=1,2,3, \ldots$ This completes the proof.

Theorem 5.4. Let $X=\left(X_{k}\right)$ be a sequence of fuzzy numbers and $\bar{d}$ be a translation invariant. Let $A=\left(a_{n k}\right)$ transform $\Gamma_{M}(F, A, p, \Delta)$ into $\Gamma_{M}(F, A, p)$. Then $\lim _{n \rightarrow \infty} a_{n k} q^{n}=0$ for all positive integers $q$.

Proof. Let

$$
t_{n}=\left(\sum_{k=1}^{\infty} a_{n k} \bar{d}\left(M\left(\frac{\left|X_{k}\right|^{1 / k}}{\rho}\right), 0\right)^{p}\right)
$$


with $\left(X_{k}\right) \in \Gamma_{M}(F, A, p, \Delta),\left(t_{n}\right) \in \Gamma_{M}(F, A, p)$ and

$$
s_{n}=\left(\sum_{k=1}^{\infty} a_{n k} \bar{d}\left(M\left(\frac{\left|X_{k}\right|^{1 / k}}{\rho}\right), 0\right)^{p}\right),
$$

$\left(s_{n}\right) \in \Gamma_{M}(F, A, p)$. Then

$$
\begin{aligned}
Y_{n} & =\left(t_{n}-s_{n}\right)=\left(\sum_{k=1}^{\infty} a_{n k} \bar{d}\left(M\left(\frac{\left|X_{k}-X_{k+1}\right|^{1 / k}}{\rho}\right), 0\right)^{p}\right) \\
& =\sum_{k=1}^{\infty} a_{n k} \bar{d}\left(M\left(\frac{\left|\Delta X_{k}\right|^{1 / k}}{\rho}\right), 0\right)^{p}
\end{aligned}
$$

and $\left(\Delta X_{k}\right) \in \Gamma_{M}(F, A, p)$ and $\left(Y_{n}\right) \in \Gamma_{M}(F, A, p)$. Hence $\left(a_{n k}\right) q^{n} \rightarrow 0$ as $n \rightarrow \infty$ for all $k$, by [13]. This completes the proof.

Theorem 5.5. Let $X=\left(X_{k}\right)$ be a sequence of fuzzy numbers and $\bar{d}$ be a translation invariant. If $A=\left(a_{n k}\right)$ transforms $\Gamma_{M}(F, A, p, \Delta)$ into $\Gamma_{M}(F, A, p, \Delta)$, then $a_{n k} q^{n} \rightarrow 0$ and $a_{n+1, k} q^{n} \rightarrow 0$ as $n \rightarrow \infty$.

Proof. From Theorems 5.2 and 5.3 we have $a_{n k} q^{n} \rightarrow 0$ and $\left(a_{n k}-a_{n+1, k}\right) q^{n}$ as $n \rightarrow \infty$ for all positive integers $q$ and for all $k$

$\Rightarrow a_{n k} q^{n} \rightarrow 0$ and $\left(a_{n k} q^{n}-a_{n+1, k} q^{n}\right) \rightarrow 0$

$\Rightarrow\left(a_{n+1, k}\right) q^{n} \rightarrow 0$ and $\left(a_{n k}\right) q^{n} \rightarrow 0$ as $n \rightarrow \infty$ for all $k$.

This completes the proof.

\section{6. $\Delta$-statistical convergence}

The idea of statistical convergence of fuzzy numbers was introduced by Nuray and Savas [34]. The generalized de la Valée-Pousin mean is defined by $t_{n}(X)=\frac{1}{\lambda_{n}} \sum_{k \in I_{n}} X_{k}$, where $\lambda=\left(\lambda_{n}\right)$ is a non-decreasing sequence of positive numbers such that $\lambda_{n+1} \leq \lambda_{n}+1, \lambda_{1}=1, \lambda_{n} \rightarrow \infty$ as $n \rightarrow \infty$ and $I_{n}=\left[n-\lambda_{n}+1, n\right]$. In this section we define this concept for the sequences.

Definition 6.1. A sequence $X=\left(X_{k}\right)$ of fuzzy numbers is said to be $\Delta$ statistial convergent to fuzzy number zero if

$$
\lim _{n \rightarrow \infty} \frac{1}{n}\left|\left\{k \leq n: \bar{d}\left(M\left(\frac{\left|\Delta X_{k}\right|^{1 / k}}{\rho}\right), 0\right) \geq \epsilon\right\}\right|=0,
$$

i.e., $\left(\bar{d}\left(M\left(\frac{\left|\Delta X_{k}\right|^{1 / k}}{\rho}\right), 0\right)\right)<\epsilon$. In this case we write

$$
S t(\Delta)-\lim _{k \rightarrow \infty}\left(M\left(\frac{\left|X_{k}\right|^{1 / k}}{\rho}\right)\right)=0 .
$$

Definition 6.2. Let $X=\left(X_{k}\right)$ be a sequence of fuzzy numbers and $p=\left(p_{k}\right)$ be a sequence of strictly positive real numbers. Then the sequence $X=\left(X_{k}\right)$ 
is said to be strongly $\Delta$-convergent if there is a fuzzy number zero such that $\lim _{n \rightarrow \infty} \frac{1}{n} \sum_{k \leq n}\left[\bar{d}\left(M\left(\frac{\left|\Delta X_{k}\right|^{1 / k}}{\rho}\right), 0\right)\right]^{p_{k}}=0$.

Definition 6.3. Let $X=\left(X_{k}\right)$ be a sequence of fuzzy numbers. Then the sequence $X=\left(X_{k}\right)$ of fuzzy numbers is said to be $\Delta$-Orlicz space of analytic if the set $\left\{M\left(\frac{\left|\Delta x_{k}\right|^{1 / k}}{\rho}: k \in N\right)\right\}$ of fuzzy numbers is Orlicz space of analytic. By $\Lambda_{M}(\Delta)$ we shall denote the set of all $\Delta$-Orlicz space of analaytic sequences of fuzzy numbers.

Theorem 6.4. If $\left(X_{k}\right),\left(Y_{k}\right) \in S t(\Delta)$ and $c \in L(\mathbb{R})$, then

(i) $S t(\Delta)-\lim \left(c\left(M\left(\frac{\left|X_{k}\right|^{1 / k}}{\rho}\right)\right)\right)=c S t(\Delta)-\lim \left(M\left(\frac{\left|X_{k}\right|^{1 / k}}{\rho}\right)\right)$,

(ii) $S t(\Delta)-\lim \left(M\left(\frac{\left|X_{k}+Y_{k}\right|^{1 / k}}{\rho}\right)\right)=S t(\Delta)-\lim \left(M\left(\frac{\left|X_{k}\right|^{1 / k}}{\rho}\right)\right)+S t(\Delta)-$ $\lim \left(M\left(\frac{\left|Y_{k}\right|^{1 / k}}{\rho}\right)\right)$, where $\bar{d}$ is a translation invariant.

Proof. (i) Let $S t(\Delta)-\lim \left(M\left(\frac{\left|X_{k}\right|^{1 / k}}{\rho}\right)\right) c \in L(\mathbb{R})$ and $\epsilon>0$ be given. Then the proof follows from the following inequality

$$
\begin{aligned}
& \frac{1}{n}\left|\left\{k \leq n: \bar{d}\left(M\left(\frac{\left|c \Delta X_{k}\right|^{1 / k}}{\rho}\right), 0\right) \geq \epsilon\right\}\right| \\
\leq & \frac{1}{n}\left|\left\{k \leq n: \bar{d}\left(M\left(\frac{\left|\Delta X_{k}\right|^{1 / k}}{\rho}\right), 0\right) \geq \frac{\epsilon}{|c|}\right\}\right| .
\end{aligned}
$$

(ii) Suppose that

$S t(\Delta)-\lim \left(M\left(\frac{\left|X_{k}\right|^{1 / k}}{\rho}\right)\right)=0$ and $S t(\Delta)-\lim \left(M\left(\frac{\left|Y_{k}\right|^{1 / k}}{\rho}\right)\right)=0$.

By Minkowski's inequality we get

$$
\begin{aligned}
& \bar{d}\left(M\left(\frac{\left|\Delta X_{k}+\Delta Y_{k}\right|^{1 / k}}{\rho}\right), 0\right) \\
= & \bar{d}\left(M\left(\frac{\left|\Delta X_{k}\right|^{1 / k}}{\rho}\right), 0\right)+\bar{d}\left(M\left(\frac{\left|\Delta Y_{k}\right|^{1 / k}}{\rho}\right), 0\right) .
\end{aligned}
$$

Therefore given $\epsilon>0$ we have

$$
\begin{aligned}
& \frac{1}{n}\left|\left\{k \leq n: \bar{d}\left(M\left(\frac{\left|\Delta X_{k}+\Delta Y_{k}\right|^{1 / k}}{\rho}\right), 0\right) \geq \epsilon\right\}\right| \\
\leq & \frac{1}{n}\left|\left\{k \leq n: \bar{d}\left(M\left(\frac{\left|\Delta X_{k}\right|^{1 / k}}{\rho}\right), 0\right) \geq \frac{\epsilon}{2}\right\}\right| \\
& +\frac{1}{n}\left|\left\{k \leq n: \bar{d}\left(M\left(\frac{\left|\Delta Y_{k}\right|^{1 / k}}{\rho}\right), 0\right) \geq \frac{\epsilon}{2}\right\}\right| .
\end{aligned}
$$


Hence $S t(\Delta)-\lim \left(M\left(\frac{\left|X_{k}+Y_{k}\right|^{1 / k}}{\rho}\right)\right)=0$. This completes the proof.

Theorem 6.5. If a sequence $X=\left(X_{k}\right)$ is $\Delta$-statistically convergent to the fuzzy number zero and $\liminf _{(n)}\left(\lambda_{n} / n\right)>0$, then it is $\Delta$-statistically convergent to zero.

Proof. Given $\epsilon>0$ we have

$$
\begin{aligned}
& \frac{1}{n}\left|\left\{k \leq n: \bar{d}\left(M\left(\frac{\left|\Delta X_{k}\right|^{1 / k}}{\rho}\right), 0\right) \geq \epsilon\right\}\right| \\
\supset & \frac{1}{n}\left|\left\{k \in I_{n}: \bar{d}\left(M\left(\frac{\left|\Delta X_{k}\right|^{1 / k}}{\rho}\right), 0\right) \geq \epsilon\right\}\right| .
\end{aligned}
$$

Therefore,

$$
\begin{aligned}
& \frac{1}{n}\left|\left\{k \leq n: \bar{d}\left(M\left(\frac{\left|\Delta X_{k}\right|^{1 / k}}{\rho}\right), 0\right) \geq \epsilon\right\}\right| \\
\geq & \frac{1}{n}\left|\left\{k \in I_{n}: \bar{d}\left(M\left(\frac{\left|\Delta X_{k}\right|^{1 / k}}{\rho}\right), 0\right) \geq \epsilon\right\}\right| \\
\geq & \frac{\lambda_{n}}{n} \frac{1}{\lambda_{n}}\left|\left\{k \in I_{n}: \bar{d}\left(M\left(\frac{\left|\Delta X_{k}\right|^{1 / k}}{\rho}\right), 0\right) \geq \epsilon\right\}\right| .
\end{aligned}
$$

Taking limit as $n \rightarrow \infty$ and using $\liminf (n)\left(\lambda_{n} / n\right)>0$, we get $X$ is $\Delta$ statistically convergent to zero. This completes the proof.

Theorem 6.6. Let $0 \leq p_{k} \leq q_{k}$ and let $\left\{\frac{q_{k}}{p_{k}}\right\}$ be bounded. Then $\Gamma_{M}(F, q, \Delta) \subset$ $\Gamma_{M}(F, p, \Delta)$.

Proof. Let

$$
X \in \Gamma_{M}(F, q, \Delta)
$$

and given $\epsilon>0$

$$
\frac{1}{n}\left|\left\{k \leq n: \bar{d}\left(M\left(\frac{\left|\Delta X_{k}\right|^{1 / k}}{\rho}\right), 0\right)^{q_{k}} \geq \epsilon\right\}\right| \rightarrow 0 \text { as } n \rightarrow \infty .
$$

Let $t_{k}=\bar{d}\left(M\left(\frac{\left|\Delta X_{k}\right|^{1 / k}}{\rho}\right), 0\right)^{q_{k}}$ and $\lambda_{k}=\frac{p_{k}}{q_{k}}$. Since $p_{k} \leq q_{k}$, we have $0 \leq$ $\lambda_{k} \leq 1$. Take $0<\lambda<\lambda_{k}$. Define $u_{k}=t_{k}\left(t_{k} \geq 1\right) ; u_{k}=0\left(t_{k}<1\right)$ and $v_{k}=$ $0\left(t_{k} \geq 1\right) ; v_{k}=t_{k}\left(t_{k}<1\right), t_{k}=u_{k}+v_{k}$, i.e., $t_{k}^{\lambda_{k}}=u_{k}^{\lambda_{k}}+v_{k}^{\lambda_{k}}$. Now it follows that

$$
u_{k}^{\lambda_{k}} \leq u_{k} \leq t_{k} \text { and } v_{k}^{\lambda_{k}} \leq v_{k}^{\lambda}
$$


Since $t_{k}^{\lambda_{k}}=u_{k}^{\lambda_{k}}+v_{k}^{\lambda_{k}}$, then $t_{k}^{\lambda_{k}} \leq t_{k}+v_{k}^{\lambda}$,

$$
\begin{aligned}
& \frac{1}{n}\left|\left\{k \leq n:\left(\bar{d}\left(M\left(\frac{\left|\Delta X_{k}\right|^{1 / k}}{\rho}\right), 0\right)^{q_{k}}\right)^{\lambda_{k}} \geq \epsilon\right\}\right| \\
\leq & \frac{1}{n}\left|\left\{k \leq n:\left(\bar{d}\left(M\left(\frac{\left|\Delta X_{k}\right|^{1 / k}}{\rho}\right), 0\right)\right)^{q_{k}} \geq \epsilon\right\}\right| \\
\Rightarrow & \frac{1}{n}\left|\left\{k \leq n:\left(\bar{d}\left(M\left(\frac{\left|\Delta X_{k}\right|^{1 / k}}{\rho}\right), 0\right)^{q_{k}}\right)^{p_{k} / q_{k}} \geq \epsilon\right\}\right| \\
\leq & \frac{1}{n}\left|\left\{k \leq n:\left(\bar{d}\left(M\left(\frac{\left|\Delta X_{k}\right|^{1 / k}}{\rho}\right), 0\right)\right)^{q_{k}} \geq \epsilon\right\}\right| \\
\Rightarrow & \frac{1}{n}\left|\left\{k \leq n:\left(\bar{d}\left(M\left(\frac{\left|\Delta X_{k}\right|^{1 / k}}{\rho}\right), 0\right)\right)^{p_{k}} \geq \epsilon\right\}\right| \\
\leq & \frac{1}{n}\left|\left\{k \leq n:\left(\bar{d}\left(M\left(\frac{\left|\Delta X_{k}\right|^{1 / k}}{\rho}\right), 0\right)\right)^{q_{k}} \geq \epsilon\right\}\right| .
\end{aligned}
$$

But $\frac{1}{n}\left|\left\{k \leq n:\left(\bar{d}\left(M\left(\frac{\left|\Delta X_{k}\right|^{1 / k}}{\rho}\right), 0\right)\right)^{q_{k}} \geq \epsilon\right\}\right| \rightarrow 0$ as $n \rightarrow \infty$ by (14). Therefore $\frac{1}{n}\left|\left\{k \leq n:\left(\bar{d}\left(M\left(\frac{\left|\Delta X_{k}\right|^{1 / k}}{\rho}\right), 0\right)\right)^{p_{k}} \geq \epsilon\right\}\right| \rightarrow 0$ as $n \rightarrow \infty$. Hence

$$
X \in \Gamma_{M}(F, p, \Delta) \text {. }
$$

From (13) and (16) we get $\Gamma_{M}(F, q, \Delta) \subset \Gamma_{M}(F, p, \Delta)$. This completes the proof.

\section{References}

[1] Y. Altin and M. Et, Generalized difference sequence spaces defined by a modulus function in a locally convex space, Soochow J. Math. 31 (2005), no. 2, 233-243.

[2] S. Aytar, Statistical limit points of sequences of fuzzy numbers, Inform. Sci. 165 (2004), no. 1-2, 129-138.

[3] M. Başarir and M. Mursaleen, Some sequence spaces of fuzzy numbers generated by infinite matrices, J. Fuzzy Math. 11 (2003), no. 3, 757-764.

[4] C. Bektas and Y. Altin, The sequence space $l_{M}(p, q, s)$ on seminormed spaces, Indian J. Pure Appl. Math. 34 (2003), no. 4, 529-534.

[5] T. Bilgin, $\Delta$-statistical and strong $\Delta$-Cesaro convergence of sequences of fuzzy numbers, Math. Commun. 8 (2003), no. 1, 95-100.

[6] H. I. Brown, The summability field of a perfect $l-l$ method of summation, J. Analyse Math. 20 (1967), 281-287.

[7] R. Colak, M. Et, and E. Malkowsky, Some topics of sequence spaces, Lecture Notes in Mathematics, Firat University Press, Elazig, Turkey, 2004.

[8] P. Diamond and P. Kloeden, Metric spaces of fuzzy sets, Fuzzy Sets and Systems 35 (1990), no. 2, 241-249.

[9] M. Et, On some topological properties of generalized difference sequence spaces, Int. J. Math. Math. Sci. 24 (2000), no. 11, 785-791. 
[10] M. Et and R. Colak, On some generalized difference sequence spaces, Soochow J. Math. 21 (1995), no. 4, 377-386.

[11] J. Fang and H. Huang, On the level convergence of a sequence of fuzzy numbers, Fuzzy Sets and Systems 147 (2004), no. 3, 417-435.

[12] H. Fast, Sur la convergence statistique, Colloquium Math. 2 (1951), 241-244.

[13] G. Fricke and R. E. Powell, A theorem on entire methods of summation, Compositio Math. 22 (1970), 253-259.

[14] J. Fridy, On statistical convergence, Analysis 5 (1985), no. 4, 301-313.

[15] C. Goffman and G. Pedrick, First Course in Functional Analysis, Prentice-Hall, Inc., Englewood Cliffs, N.J., 1965.

[16] M. Işik, On statistical convergence of generalized difference sequences, Soochow J. Math. 30 (2004), no. 2, 197-205.

[17] P. K. Kamthan, Bases in a certain class of a Frechet space, Tamkang J. Math. 7 (1976), no. 1, 41-49.

[18] P. K. Kamthan and M. Gupta, Sequence Spaces and Series, Lecture Notes in Pure and Applied Mathematics, 65. Marcel Dekker, Inc., New York, 1981.

[19] H. Kizmaz, On certain sequence spaces, Canad. Math. Bull. 24 (1981), no. 2, 169-176.

[20] M. A. Krasnosel'skii and Ja. B. Rutickii, Convex Functions and Orlicz Spaces, Translated from the first Russian edition by Leo F. Boron. P. Noordhoff Ltd., Groningen 1961.

[21] J. S. Kwon, On statistical and p-Cesaro convergence of fuzzy numbers, Korean J. Comput. Appl. Math. 7 (2000), no. 1, 195-203.

[22] L. Leindler, Über die verallgemeinerte de la Vallée-Poussinsche Summierbarkeit allgemeiner Orthogonalreihen, Acta Math. Acad. Sci. Hungar. 16 (1965), 375-387.

[23] J. Lindenstrauss and L. Tzafriri, On Orlicz sequence spaces, Israel J. Math. 10 (1971), 379-390.

[24] I. J. Maddox, Elements of Functional Analysis, Cambridge University Press, LondonNew York, 1970.

[25] _ Sequence spaces defined by a modulus, Math. Proc. Cambridge Philos. Soc. 100 (1986), no. 1, 161-166.

[26] M. Matloka, Sequences of fuzzy numbers, Busefal 28 (1986), 28-37.

[27] E. T. Mikail and F. Nuray, $\Delta^{m}$-statistical convergence, Indian J. Pure Appl. Math. 32 (2001), no. 6, 961-969.

[28] M. Mursaleen and M. Başarir, On some new sequence spaces of fuzzy numbers, Indian J. Pure Appl. Math. 34 (2003), no. 9, 1351-1357.

[29] M. Mursaleen, M. A. Khan, and Qamaruddin, Difference sequence spaces defined by Orlicz functions, Demonstratio Math. 32 (1999), no. 1, 145-150.

[30] H. Nakano, Concave modulars, J. Math. Soc. Japan 5 (1953), 29-49.

[31] S. Nanda, On sequences of fuzzy numbers, Fuzzy Sets and Systems 33 (1989), no. 1, $123-126$.

[32] I. Niven and H. S. Zuckerman, An Introduction to the Theory of Numbers, Fourth ed., John Wiley and Sons, New York, 1980.

[33] F. Nuray, Lacunary statistical convergence of sequences of fuzzy numbers, Fuzzy Sets and Systems 99 (1998), no. 3, 353-355.

[34] F. Nuray and E. Savaş, Statistical convergence of sequences of fuzzy numbers, Math. Slovaca 45 (1995), no. 3, 269-273.

[35] W. Orlicz, b̈er Raume $\left(L^{M}\right)$, Bull. Internat. Acad. Polon. Sci. Lett. Cl. Sci. Math. Nat. Ser. A (1936), 93-107.

[36] S. D. Parashar and B. Choudhary, Sequence spaces defined by Orlicz functions, Indian J. Pure Appl. Math. 25 (1994), no. 4, 419-428.

[37] K. Chandrasekhara Rao and N. Subramanian, The Orlicz space of entire sequences, Int. J. Math. Math. Sci. 2004 (2004), no. 65-68, 3755-3764. 
[38] K. Chandrasekhara Rao and T. G. Srinivasalu, Matrix operators on analytic and entire sequences, Bull. Malaysian Math. Soc. (2) 14 (1991), no. 1, 41-54.

[39] W. H. Ruckle, FK spaces in which the sequence of coordinate vectors is bounded, Canad. J. Math. 25 (1973), 973-978.

[40] E. Savaş, On strongly $\lambda$-summable sequences of fuzzy numbers, Inform. Sci. 125 (2000), no. $1-4,181-186$

[41] I. J. Schoenberg, The integrability of certain functions and related summability methods, Amer. Math. Monthly 66 (1959), 361-375.

[42] B. C. Tripathy, M. Et, and Y. Altin, Generalized difference sequence spaces defined by Orlicz function in a locally convex space, J. Anal. Appl. 1 (2003), no. 3, 175-192.

[43] A. Wilansky, Summability through Functional Analysis, North-Holland Mathematics Studies, 85. Notas de Matematica [Mathematical Notes], 91. North-Holland Publishing Co., Amsterdam, 1984.

[44] C. Wu and G. Wang, Convergence of sequences of fuzzy numbers and fixed point theorems for increasing fuzzy mappings and application, Fuzzy Sets and Systems 130 (2002), no. 3, 383-390.

[45] L. A. Zadeh, Fuzzy sets, Information and Control 8 (1965), 338-353.

Nagarajan Subramanian

Department of Mathematics

SASTRA UNIVERSITY

TANJORE-613 401, INDIA

E-mail address: nsmaths@yahoo.com

AYHAN Esi

Department of Mathematics

SCIENCE AND ART FACUlTy

ADIYAMAN UNIVERSITY

Adiyaman 02040, Turkey

E-mail address: aesi23@hotmail.com 